\title{
Garbage Collection Without Paging
}

\author{
Matthew Hertz, Yi Feng, and Emery D. Berger \\ Department of Computer Science \\ University of Massachusetts \\ Amherst, MA 01003 \\ \{hertz, yifeng, emery\}@cs.umass.edu
}

\begin{abstract}
Garbage collection offers numerous software engineering advantages, but it interacts poorly with virtual memory managers. Most existing garbage collectors visit many more pages than the application itself and touch pages without regard to which ones are in memory, especially during full-heap garbage collection. The resulting paging can cause throughput to plummet and pause times to spike up to seconds or even minutes. We present a garbage collector that avoids paging. This bookmarking collector cooperates with the virtual memory manager to guide its eviction decisions. It records summary information ("bookmarks") about evicted pages that enables it to perform in-memory full-heap collections. In the absence of memory pressure, the bookmarking collector matches the throughput of the best collector we tested while running in smaller heaps. In the face of memory pressure, it improves throughput by up to a factor of five and reduces pause times by up to a factor of 45 over the next best collector. Compared to a collector that consistently provides high throughput (generational mark-sweep), the bookmarking collector reduces pause times by up to $218 \mathrm{x}$ and improves throughput by up to $41 \mathrm{x}$.
\end{abstract}

\section{Introduction}

Garbage collection is a primary reason for the popularity of languages like Java and C\# [47, 54]. However, garbage collection requires considerably more space than explicit memory management $[58,32]$. The result is that fewer garbage-collected applications can fit in a given amount of RAM. If even one garbagecollected application does not fit in available physical memory, the garbage collector will induce paging, or traffic between main memory and the disk. Because disk accesses are approximately six orders of magnitude more expensive than main memory accesses, paging significantly degrades performance. Paging can also lead to pause times lasting for tens of seconds or minutes.

Even when an application's working set fits in main memory, the act of garbage collection may induce paging. During major (fullheap) collections, most existing garbage collectors touch pages without regard to which pages are resident in memory and visit many more pages than those in the application's working set. This action also disrupts information about the reference history tracked by the

Permission to make digital or hard copies of all or part of this work for personal or classroom use is granted without fee provided that copies are not made or distributed for profit or commercial advantage and that copies bear this notice and the full citation on the first page. To copy otherwise, to republish, to post on servers or to redistribute to lists, requires prior specific permission and/or a fee.

Submitted to PLDI 2005, Chicago, Illinois

Copyright 2005 ACM 1-11111-111-1/11/1111 ..\$5.00 virtual memory manager.

While this phenomenon is widely known, previous work has attacked it only indirectly. For example, generational garbage collectors concentrate their collection efforts on short-lived objects [38, 51]. Because these objects have a low survival rate, generational collection reduces the frequency of full heap garbage collections. However, when a generational collector eventually performs a fullheap collection, it triggers paging.

This problem has led to a number of workarounds. One standard way to avoid paging is to size the heap so that it never exceeds the size of available physical memory. However, choosing an appropriate size statically is impossible on a multiprogrammed system, where the amount of available memory changes. Another possible approach is to overprovision systems with memory, but high-speed, high-density RAM remains expensive. It is often impractical to require that users purchase more memory in order to run garbagecollected applications. Even in an overprovisioned system, because garbage collectors perform so poorly in the face of memory pressure, just one unanticipated workload exceeding available memory can render a system unresponsive. This performance hit has led some to recommend that garbage collection not be used at all, except for very small applications [48].

Contributions: This paper introduces bookmarking collection (BC), a garbage collection algorithm that virtually eliminates garbage collector-induced paging. Bookmarking collection records summary information ("bookmarks") about outgoing pointers from pages that have been evicted to disk. Once a page is evicted from memory, BC does not touch it unless the application itself makes it resident. Instead of visiting evicted pages, BC uses bookmarks to assist in garbage collection. These bookmarks, together with BC's heap organization and cooperation with the virtual memory manager, allow BC to perform full-heap, compacting garbage collection without paging, even when large portions of the heap have been evicted.

We have implemented BC in Jikes RVM [7, 8] using the MMTk toolkit [19]. The bookmarking collector relies on some additional operating system support, which we have implemented as a modest extension to the Linux virtual memory manager (approximately six hundred lines of code). Without memory pressure, BC's performance matches that of generational mark-sweep (GenMS), a collector that consistently provides high throughput [18]. Under memory pressure, bookmarking collection outperforms the next best garbage collector we tested by a factor of five, and reduces pause times by a factor of 45 . Compared to GenMS, bookmarking collection yields up to a 218 -fold reduction in pause times and improves throughput by up to $41 \mathrm{x}$. Bookmarking thus provides greater utilization of available memory than other collectors.

The paper is organized as follows: Section 2 provides an overview 
of bookmarking collection, and Section 3 describes the bookmarking garbage collector in detail. Section 4 discusses the limits of bookmarking collection and implications of our design decisions. Section 5 presents some key implementation details, including our extensions to the Linux virtual memory manager. Section 6 presents empirical results comparing the performance of bookmarking collection to a range of existing garbage collectors, both with and without memory pressure. Section 7 discusses related work, Section 8 presents directions for future work, and Section 9 concludes.

\section{Overview}

The bookmarking collector was designed to meet three goals: low space consumption, high throughput, and the elimination of all GC-induced page faults. BC minimizes space consumption by using mark-sweep collection and performing compaction when under memory pressure. It provides high throughput by using a nursery generation to manage short-lived objects. Finally, and most importantly, it organizes the heap into groups of pages and reacts to signals from the virtual memory manager whenever pages are scheduled for eviction to disk or made resident in main memory.

The performance of existing garbage collectors suffers under memory pressure because the collector visits all reachable objects, including those on evicted pages. $\mathrm{BC}$ avoids this by scanning pages prior to eviction and remembering their outgoing pointers by "bookmarking" targeted objects. When BC is notified of an impending eviction and cannot shrink the heap or discard an empty page, BC selects an appropriate victim page (with live data). BC scans each object on the page, bookmarks the target of any references, and increments a counter in the target superpage's header. Once BC has processed all of the objects, it informs the virtual memory manager that the page can be evicted. Afterwards, $\mathrm{BC}$ will not visit this page again and can continue to collect the heap without paging.

\section{Bookmarking Collection}

The bookmarking collector is a generational collector with a bumppointer nursery, a compacting mature space, and a page-based large object space. $\mathrm{BC}$ divides the mature space into superpages, pagealigned groups of four contiguous pages (16K). BC manages mature objects using segregated size classes $[12,13,16,37,39,44$, 55]: objects of different sizes are allocated onto different superpages. Completely empty superpages can be reassigned to any size class $[16,39]$.

BC uses size classes designed to minimize both internal and external fragmentation (which we bound at 25\%). Each allocation size up to 64 bytes has its own size class. Larger object sizes fall into a range of 37 size classes; for all but the largest five, these have a worst-case internal fragmentation of $15 \%$. The five largest classes have between $16 \%$ and $33 \%$ worst-case internal fragmentation; we cannot do better without violating our bound on external fragmentation. BC allocates objects larger than 8180 bytes (half the size of a superpage minus metadata) into the large object space.

When the heap fills, BC typically performs mark-sweep garbage collection. We use mark-sweep for two reasons. First, it provides good program throughput and short GC pauses. More importantly, mark-sweep does not increase memory pressure by needing a copy reserve of pages. ${ }^{1}$

\subsection{Managing Remembered Sets}

Like all generational collectors, BC needs a method of remembering pointers from the older to the younger generation. It normally stores these pointers in page-sized write buffers, which are fast to

\footnotetext{
${ }^{1}$ Usually this copy reserve is half the heap size, but Sachindran et al. present copying collectors that allow the use of much smaller copy reserves [45, 46].
}

store and process but may demand unbounded amounts of space. To limit this space overhead, BC processes buffers when they fill. During this processing, it removes entries for pointers from the mature space and instead marks a card for the source object in the card table used during the marking phase of garbage collection. $\mathrm{BC}$ begins each nursery collection by reviewing these cards and scanning objects whose cards are marked. After compacting the remaining entries, the filtered slots then become available for future storage. This filtering allows the bookmarking collector to use the fast processing of write buffers but often consumes only a single page.

\subsection{Compacting Collection}

Because mark-sweep collection does not compact the heap, fragmentation can cause it to increase memory pressure. Using marksweep, the bookmarking collector cannot reclaim a superpage if it contains just one reachable object. Whenever a full garbage collection does not free enough pages to satisfy the current allocation request, $\mathrm{BC}$ performs a two-pass compacting collection.

$\mathrm{BC}$ begins this compacting collection with a marking phase. Each time it marks an object, it also increments a counter for the object's size class. After marking, BC computes the minimum number of superpages needed to hold the marked objects for each size class. It then selects a minimum set of "target" superpages that contain enough space to hold all of the marked objects (the target also includes pages with bookmarked objects). It then copies objects in a single pass with a Cheney scan. BC checks if visited objects are on target superpages. BC forwards objects not on target superpages, while leaving those objects already on a target in place. After compaction, reachable objects are only on target superpages and the bookmarking collector can therefore free all non-target (garbage) superpages.

\subsection{Interaction with the Virtual Memory Manager}

The approach described so far allows BC to avoid increasing memory pressure during garbage collection. In the face of paging, $\mathrm{BC}$ cooperates with the virtual memory manager to adapt to changing memory pressure by reducing the size of the heap. It uses its knowledge of the heap to make good paging decisions. We extend the virtual memory manager so that it can cooperate with the collector; we describe these extensions in Section 5.1. Communication with the virtual memory manager occurs only under memory pressure.

The virtual memory manager notifies $\mathrm{BC}$ whenever a page is scheduled for eviction or made resident. BC then updates a bit array that records which pages are resident. Whenever $\mathrm{BC}$ allocates a new superpage, it checks in the bit array if the pages are already resident. If they are not, it increases its estimate of the current footprint and records that these pages are now in-core. During tracing, the collector uses the bit array to avoid following pointers into pages that are not resident.

\section{Discarding Empty Pages}

When the virtual memory manager signals the pending eviction of a page, $\mathrm{BC}$ scans the bit array for an empty, but resident, page. If it can find such a discardable page, $\mathrm{BC}$ directs the virtual memory manager to reclaim it. When a discardable page cannot be found, $\mathrm{BC}$ triggers a collection and afterward directs the virtual memory manager to discard one of the newly-emptied pages (if one exists). This functionality already exists in most operating systems (e.g., Linux and Solaris) in the form of the madvise system call with the MADV_DONTNEED flag.

\section{Keeping the Heap Memory-Resident}

The notification of a pending eviction also alerts BC that the current heap footprint is slightly larger than available memory. Unlike 
previous collectors, $\mathrm{BC}$ will not grow at the expense of paging, but instead tries limiting the heap to its current footprint. If memory pressure continues to increase, $\mathrm{BC}$ discards additional empty pages and uses this new estimate as the target footprint. BC shrinks the heap to keep it entirely in-core and thus avoid incurring the cost of a page fault. Because BC cannot guarantee that growing the heap will not cause more heap pages to be scheduled for eviction, it increases the heap size only when needed for the program to complete.

\section{Bookmarking}

When a non-empty heap page must be evicted, BC first chooses an appropriate victim page. The page proposed by the virtual memory manager is usually an appropriate victim, since it is likely to be the least-recently used page. However, BC will not select a page that it knows will soon be used, such as pages in the nursery or containing needed metadata (including superpage headers, which we discuss below). BC touches such proposed victim pages to prevent their eviction. This causes a different victim page to be scheduled for eviction.

If a garbage collection does not create discardable pages, BC scans the victim page for outgoing pointers and bookmarks the target objects of these outgoing references. These bookmarks (a single bit stolen from the status word in the object header) act as a secondary set of roots, allowing full memory-resident collections without accessing evicted pages and thus without causing any page faults.

In addition to setting the bookmark bit for each target object on a superpage, $\mathrm{BC}$ increments the incoming bookmark counter for the targeted superpage. BC uses this counter (the number of evicted pages pointing to objects on a given superpage) to release bookmarks when incoming pages become resident (we discuss bookmark clearing in Section 3.3).

BC stores this superpage metadata directly in the superpage header, which means that it must keep the superpage headers resident. This metadata is needed for both allocation and GC, so $O(1)$ access is important. Storing it off to the side would create a large pool of unevictable pages, including information for superpages that do not exist. While storing the metadata in the superpage header means we cannot evict one-fourth of the pages, it simplifies memory layout (and corresponding eviction/reloading code) and speeds access to the metadata.

Since superpage headers are always resident, BC can increment a superpage's incoming bookmark counter without a page fault. However, it cannot always bookmark every target, since these may be on evicted pages. BC therefore conservatively bookmarks every object on a page before it is evicted.

After bookmarking is complete, the victim page can safely be evicted. Having just been touched, however, the virtual memory manager would not normally schedule it for eviction. BC thus communicates with the virtual memory manager one more time, informing it that this page should be evicted. The stock Linux virtual memory manager does not provide support for this kind of operation, so we added a vm_relinquish () system call. This call allows user processes to voluntarily surrender a list of pages. These pages are placed at the end of the inactive queue and are quickly swapped out.

\section{Collection After Bookmarking}

$\mathrm{BC}$ begins the marking phase of subsequent full-heap garbage collections by scanning its superpages and large object space for memoryresident bookmarked objects. While expensive, the cost of scanning every object is often much smaller than the cost of even a single page fault. BC further reduces the cost of this scanning by scanning only the superpages whose incoming bookmark count is nonzero. During this scan, BC marks and processes bookmarked objects as if they were root-referenced. Once BC completes this scan, it has remembered all the references on evicted objects and can conservatively complete the collection without touching evicted pages. BC now follows its usual marking phase, but ignores references to evicted objects. Once marking completes, reachable objects are either marked or evicted. Finally, BC sweeps the memoryresident pages to complete collection.

When compaction is triggered, only slightly more processing is needed. After marking the heap, BC updates the marked counts to reserve space for every possible object on evicted pages. When selecting target superpages, BC first selects all superpages containing bookmarked objects or evicted pages, and then selects other superpages, as needed.

$\mathrm{BC}$ also adds a scan to its copying phase to find and process bookmarked objects. BC never moves evicted objects, since they reside on target superpages. BC also will not copy bookmarked objects and will not update (evicted) pointers to the object.

\section{Clearing Bookmarks}

$\mathrm{BC}$ largely eliminates page faults caused by the garbage collector, but it cannot prevent mutator page faults. BC protects evicted pages so that it receives a signal whenever a heap page is faulted in. Because the notification arrives before the page can be accessed by the mutator, $\mathrm{BC}$ can remove the bookmarks created when the page was evicted.

When a page is brought back into main memory, BC scans each object and decrements the incoming bookmark counter for referenced objects' superpages. Superpages whose bookmark counters are reduced to zero no longer contain any objects referred to from evicted objects. Since all references to the object can now be found during collection, $\mathrm{BC}$ clears all the bookmarks on that superpage. If the page just paged in also has a zero incoming bookmark count, $\mathrm{BC}$ clears all of the page's bookmarks, including those that were conservatively set when the page was evicted.

\section{Complications}

So far, we have described page eviction as if the kernel schedules evictions on a page-by-page basis and maintains a constant number of pages in memory. In fact, the virtual memory manager schedules pages for eviction in large batches in order to hide the latency of disk accesses. As a result, the size of available memory can fluctuate suddenly. The virtual memory manager also operates asynchronously from the collector, meaning that it can run ahead of the collector and evict a page before $\mathrm{BC}$ has a chance to process it.

To avoid this problem, BC maintains a reserve of empty pages and begins a collection when these are the only discardable pages left. If, during garbage collection, the virtual memory manager schedules pages for eviction, $\mathrm{BC}$ discards pages from this reserve while recording the pages actually scheduled for eviction. If the collection does not free enough pages to replenish the cache of discardable pages, $\mathrm{BC}$ examines the pages previously scheduled for eviction and, if they are not empty, processes and evicts them. This preventive bookmarking ensures $\mathrm{BC}$ always keeps some pages in memory in which to allocate objects and ensures BC has time to process pages before their eviction.

\section{Discussion}

The key principle behind bookmarking collection is that the garbage collector must avoid touching evicted pages. A natural question is whether it is possible to perform complete garbage collection (reclaiming all unreachable objects) without paging in any evicted pages. The answer to this question is negative. 
In general, it is impossible to perform complete garbage collection without traversing non-resident objects. Consider the case when we must evict a pageful of one-word objects that all point to objects on other pages. A lossless summary of reachability information for this page requires as much memory as the objects themselves, which we no longer have room for.

This limitation means that, in the absence of other information about reachability, we must rely on conservative summaries of connectivity information. Possible summaries include summarizing pointers on a page-by-page basis, compressing pointers in memory, and maintaining reference counts. To minimize the space and processing required for summaries, we chose the most parsimonious summarization heuristic: a single bookmark bit, stolen from the object's header. If an object has this bit on, we know that it may be the target of at least one pointer from an evicted page.

While this summary information is "free", there is a potential space cost to bookmarking. Because BC must target all superpages containing bookmarked objects and evicted pages, compacting collection cannot always minimize the size of the heap. BC also must treat all bookmarked objects as reachable, even though they may be garbage.

Despite these apparent costs, we have found that bookmarking does not substantially increase the minimum heap size that $\mathrm{BC}$ requires. We show in Section 6.3 that even in the face of megabytes of evicted pages, BC continues to run in very tight heap sizes. Such tight heaps mean that $\mathrm{BC}$ will perform more frequent garbage collections, but the time needed for these collections is still far less than the cost of the page faults that would otherwise occur.

In the event that the heap is exhausted, BC preserves completeness by performing a full heap garbage collection (touching evicted pages). Note that this worst-case situation for bookmarking collection (which we have yet to observe) is the common case for existing garbage collectors. We show in Section 6 that BC's approach effectively eliminates collector-induced paging.

\section{Implementation Details}

We implemented the bookmarking collector using MMTk [19] and Jikes RVM version 2.3.2 [7, 8]. When implementing the BC algorithm within MMTk and Jikes, we needed to make two minor modifications. In Jikes, object headers for scalars are found at the end of the object while object headers for arrays are placed at the start of an object. ${ }^{2}$ This placement is useful for optimizing NULL checks [7], but makes it difficult for BC to find object headers when scanning pages. We solve this problem by further segmenting our allocation to allow superpages to hold either only scalars or only arrays. BC stores the type of objects contained in the superpage within each superpage header. Using this type and size class information in the superpage header (accessed by bit-masking), BC can quickly locate all objects on each page.

$\mathrm{BC}$ does not employ two of the object layout optimizations included in Jikes RVM. Jikes normally aligns objects along word (4byte) boundaries, but allocates objects or arrays containing longs or doubles on an 8-byte boundary. Aligning data this way improves performance, but requires $\mathrm{BC}$ to know an object's type to find its header. Another optimization allows Jikes RVM to compute an object's hash code based upon its address. While this provides many benefits, address-based hashing also requires that copied objects grow by one word, disrupting the size-class object counts that $\mathrm{BC}$ maintains.

While we needed to remove these optimizations from BC builds, we did not want to bias our results by removing them from builds

\footnotetext{
${ }^{2}$ In a just-released version of Jikes RVM, all headers start at the beginning of the object.
}

that would benefit. We therefore include these optimizations for all builds except BC.

\subsection{Kernel Support}

The bookmarking collector improves garbage collection paging performance primarily by cooperating with the virtual memory manager. We extended the Linux kernel to enable this cooperative garbage collection. This extension consists of changes or additions to approximately six hundred lines of code (excluding comments), as measured by SLOCcount [53].

We implemented our modifications on the 2.4.20 Linux kernel. This kernel uses an approximate global LRU replacement algorithm. User pages are either kept in the active list (managed by the clock algorithm) or the inactive list (a FIFO queue). Pages are evicted from the end of the inactive list.

When the garbage collector begins, it registers itself with the operating system so that it will receive notification of paging events. The kernel will then notify the runtime system when a page is initially scheduled for eviction. The signal from the kernel includes the address of the relevant page.

To maintain information about process ownership of pages, we applied Scott Kaplan's lightweight version of Rik van Riel's reverse mapping patch $[35,52]$. This patch allows the kernel to determine the owning process only of pages currently in physical memory. We extended this reverse mapping to include pages on the swap partition (evicted to disk), and extended the mincore call to work on heap pages. These extensions account for almost one-third of the lines of code that we added to the kernel.

$\mathrm{BC}$ needs timely memory residency information from the virtual memory manager. To ensure the timeliness of this communication, we implemented our notifications using Linux real-time signals. Real-time signals in the Linux kernel are queueable. Unlike other notification methods, we can use these signals without worrying about signals lost due to other process activity.

\section{Results}

We evaluated the performance of BC by comparing it to five garbage collectors included with Jikes RVM: MarkSweep, SemiSpace, GenCopy, GenMS (Appel-style generational collectors using bumppointer and mark-sweep mature spaces, respectively), and CopyMS (a variant of GenMS which performs only whole heap garbage collections). We intended to include $M C^{2}$ [46] and GenRC (ulterior reference counting [20]) in this study, but these collectors are not stable on the current version of MMTk. We hope to report on these collectors in the final version of this paper.

\begin{tabular}{|lcc|}
\hline \multicolumn{3}{|c|}{ Benchmark statistics } \\
Benchmark & Total Bytes Alloc & Min. Heap \\
\hline & SPECjvm 98 \\
201_compress & $109,190,172$ & $16,777,216$ \\
202_jess & $267,602,628$ & $12,582,912$ \\
205_raytrace & $92,381,448$ & $14,680,064$ \\
209_db & $61,216,580$ & $19,922,944$ \\
213_javac & $181,468,984$ & $19,922,944$ \\
228_jack & $250,486,124$ & $11,534,336$ \\
\hline \multirow{3}{*}{ DaCapo } \\
ipsixql & $350,889,840$ & $11,534,336$ \\
jython & $770,632,824$ & $11,534,336$ \\
\hline \multicolumn{3}{|c}{ SPECjbb2000 } \\
pseudoJBB & $233,172,290$ & $35,651,584$ \\
\hline
\end{tabular}

Table 1: Memory usage statistics for our benchmark suite. 
Table 1 describes our benchmarks, which include the SPECjvm98 benchmark suite [28], pseudoJBB (a fixed-workload variant of SPECjbb [27]), and two applications from the DaCapo benchmark suite, ipsixql and jython. We compare execution and pause times of the pseudoJBB benchmark on our extended Linux kernel. This benchmark is widely considered to be the most representative of a server workload (for example, Adl-Tabatabai et al.'s PLDI 2004 paper [5] also relies on it) and is the only one of our benchmarks with a significant memory footprint.

\subsection{Methodology}

We performed all measurements on a $1.6 \mathrm{GHz}$ Pentium M Linux machine with $1 \mathrm{~GB}$ of RAM and $2 \mathrm{~GB}$ of local swap space. This processor includes a $32 \mathrm{~KB} \mathrm{~L} 1$ data cache and a $1 \mathrm{MB} \mathrm{L} 2$ cache. We report the mean of five runs with the system in single-user mode and the network disabled.

To duplicate the environment found in live servers, we compile all of the code using the optimizing compiler. We did not want compilation to affect our end results, however, as servers rarely spend time compiling code. We follow Bacon et al. [13] and run two iterations of each benchmark, but report results only from the second iteration. The first iteration optimizes all of the code. Because compilation allocates onto the heap, the heap size is allowed to change during this iteration. After the first iteration is complete, we perform a full heap collection to remove compilation objects and data remaining from the first run. We then measure the second iteration of the benchmark. Using this "compile-and-reset" methodology, we can compare the performance of collectors in an environment similar to that found in a large server.

To examine the effects of memory pressure on garbage collection paging behavior, we simulate increasing memory pressure caused by another application starting up or requesting memory. We begin these experiments by making available only enough memory for Jikes to complete the compilation phase without any memory pressure. We also use an external process we call signalmem. Jikes RVM notifies signalmem when it completes the first iteration of a benchmark. Once alerted, signalmem allocates a large array using mmap and begins to touch pages and then uses mlock to pin them in-core. The initial amount of memory pinned, the total amount of memory signalmem pinned, and the rate at which this memory is pinned are specified via command-line parameters. Using signalmem provides repeatable measurements under memory pressure while reducing disruption due to CPU load. This approach also allows us to compare the effects of different levels of memory pressure and a variety of page evictions rates.

\subsection{Performance Without Memory Pressure}

While the key goal of bookmarking collection is to avoid paging, it would not be practical if it did not provide competitive throughput in the absence of memory pressure. Figure 1 summarizes the results for the case when there is sufficient memory to run the benchmarks without any paging. We present the geometric mean increase in execution time relative to $\mathrm{BC}$ at each relative heap size for each collector.

As expected, $\mathrm{BC}$ is closest in performance to GenMS, although $\mathrm{BC}$ runs at smaller heap sizes. Both collectors perform nursery collection and have a segregated-fit mark-sweep mature space, and behave similarly at large heap sizes and without memory pressure. At the largest heap size (where heap compaction is not needed) the two collectors are virtually tied (BC runs $0.3 \%$ faster). However, at smaller sizes, BC's compaction allows it to run in smaller heaps. For example, $\mathrm{BC}$ runs $4 \%$ faster at the $1.25 \mathrm{x}$ heap size.

The next best collector is GenCopy, which runs as fast as BC at the largest heap sizes but averages 7\% slower at heaps as large as

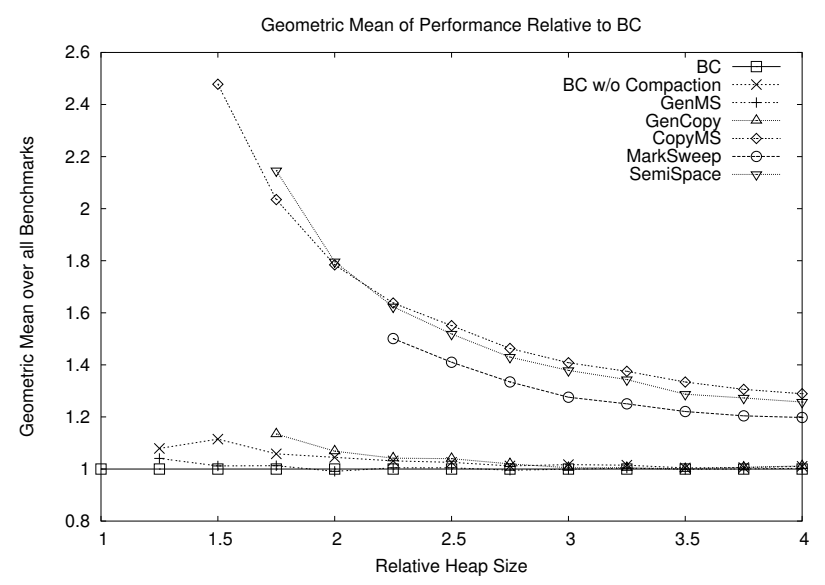

Figure 1: Geometric mean of execution time relative to $\mathrm{BC}$ absent memory pressure and across all benchmarks. At the smaller sizes, heap compaction allows $\mathrm{BC}$ to require less space while providing the best performance. Compaction is not needed at larger heap sizes, but BC typically continues to provide high performance.

twice the minimum. The fact that GenCopy generally does not exceed BC's performance suggests that BC's segregated size classes do not significantly impact locality. Unsurprisingly, BC's performance is much greater than the single-generation collectors. At the largest heap size, MarkSweep averages a 20\% and CopyMS a 29\% slowdown. No collector at any heap size performs better on average than $\mathrm{BC}$, demonstrating that the $\mathrm{BC}$ provides high performance when memory pressure is low.

\subsection{Performance Under Memory Pressure}

We evaluate the impact of memory pressure using three sets of experiments. We first measure the effect of steady memory pressure. Next, we measure the effect of dynamically growing memory pressure, as would be caused by the creation of another process or a rapid increase in demand (e.g., the "Slashdot effect"). Finally, we run multiple JVMs simultaneously. We evaluate $\mathrm{BC}$ with the pseudoJBB benchmark, which is the benchmark most representative of a server workload and one that has a significant memory footprint.

\section{Steady Memory Pressure}

To examine the effects of running under steady memory pressure, we measured the available memory needed at each heap size to run all of the collectors without evicting a page. Starting with that amount of available memory, we began the second iteration by having signalmem remove memory equal to $60 \%$ of the heap size. Results of these experiments are shown in Figure 2. Note that we do not show results for MarkSweep in these graphs, because runs with this collector take hours to complete.

Figure 2 shows that under steady memory pressure, BC outperforms most of the other collectors (and all of the generational collectors). Although SemiSpace outperforms BC at the 80-95MB heap sizes, its execution time goes off the chart soon after. CopyMS also outperforms BC in the same range of heap sizes but runs nearly twice as slow as BC at the 130MB heap size. At this size, GenMS's average pause time is also 30 times longer (around 3 seconds). To test CopyMS's behavior under greater memory pressure, we measured the effect of removing memory equal to $70 \%$ of the heap size. We found that it can take CopyMS over an hour to execute 


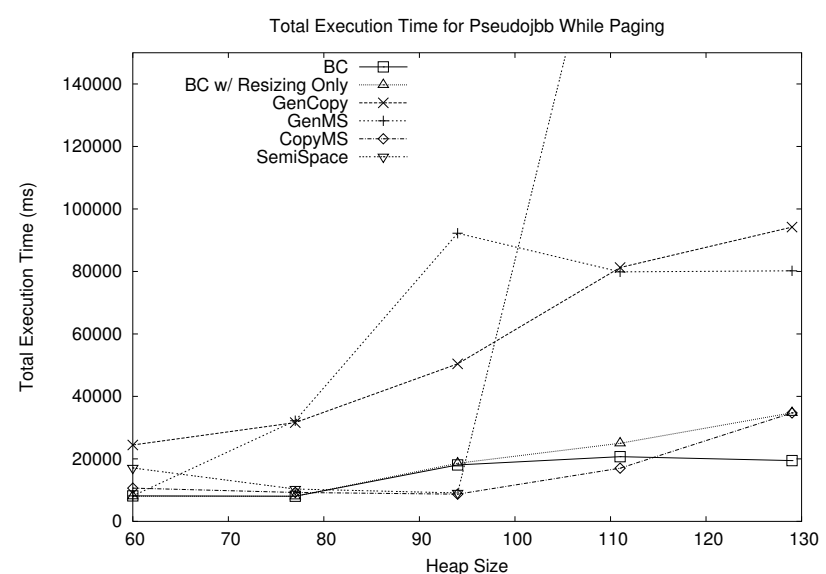

(a) Execution time running pseudoJBB.

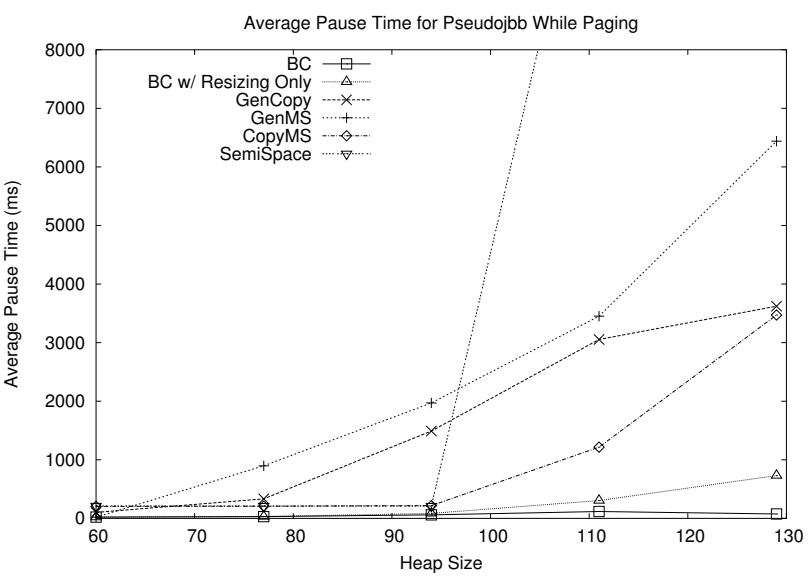

(b) Average GC pause time running pseudoJBB.

Figure 2: Steady memory pressure (increasing from left to right), where available memory is sufficient to hold only $40 \%$ of the heap. As the heap becomes tighter, BC runs 7 to 8 times faster than GenMS and in less than half the time needed by CopyMS. Bookmarking is faster and yields shorter pause times than simply resizing the heap.

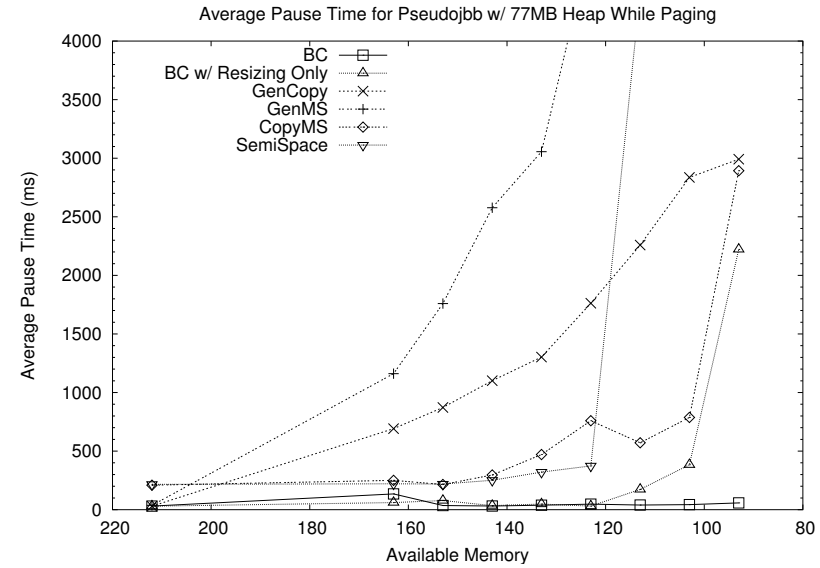

Figure 3: Dynamic memory pressure (increasing from left to right): average GC pause time running pseudoJBB. BC's average pause times remain unaffected by increasing memory pressure.

pseudoJBB, while BC's performance remains largely unchanged.

\section{Dynamic Memory Pressure}

To simulate a spike in memory pressure, we invoked signalmem so that it initially allocates $30 \mathrm{MB}$ and then allocates additional memory at a rate of $1 \mathrm{MB}$ every $100 \mathrm{~ms}$ until it reaches the desired level of available memory. Figure 3 shows the average pause times and Figure 4 shows the average execution time for pseudoJBB as memory pressure increases (i.e., as available memory shrinks).

Figures 3 and 4 show that under memory pressure, BC significantly outperforms all of the other collectors both in total execution time and pause times. (As above, we do not present MarkSweep here because each run takes hours to complete.) Because the mark-sweep based collectors do not perform compaction, objects become spread out over a range of pages. Visiting these pages during a collection can trigger a cascade of page faults that results in the orders-of-magnitude increases in execution time for MarkSweep and GenMS. For instance, at the largest heap size, GenMS's average garbage collection pause takes nearly 10 seconds - longer than it needed to execute pseudoJBB without memory pressure. Even when collections are relatively rare, spreading objects across a large number of pages can cause an increase in mutator faults.

Compacting objects onto fewer pages can reduce faults, but the copying collectors also suffer from paging effects. For example, the execution times for GenCopy shown in Figure 4(a) are an order of magnitude larger than its times when not paging. Paging also increases the average GenCopy pause to several seconds, while BC's pause times remain largely unchanged.

The collectors that come closest in execution time to BC in Figure 4 are SemiSpace and CopyMS, which perform well at low to moderate memory pressure, although they perform far worse both under no memory pressure and under severe memory pressure. This is due to pseudoJBB's allocation behavior. pseudoJBB initially allocates a few immortal objects and then allocates only short-lived objects. While these collectors reserve heap space to copy the survivors of a collection, little of this space is used. LRU ordering causes nursery pages filled with dead objects to be evicted. While SemiSpace ultimately reuses these pages in subsequent collections, CopyMS's mark-sweep mature object space allows better heap utilization and fewer collections. This delays paging, but does not prevent it.

We next present mutator utilization curves, following the methodology of Cheng and Blelloch [24]. They define mutator utilization as the fraction of time that the mutator runs during a given time window. Rather than presenting minimum mutator utilization (MMU) for each window, which can lead to a larger window size having a lower utilization than a smaller one, we adopt the methodology of Sachindran et al. and instead present bounded mutator utilization, or BMU [46]. The BMU for a given window size is the minimum mutator utilization for all windows of that size or greater.

Figure 5 shows the BMU curves for the dynamic memory pressure experiments. With moderate memory pressure (143MB available RAM), both variants of BC and MarkSweep do very well, but all of the other collectors exhibit poorer utilization. In particular, GenMS requires window sizes orders of magnitude larger than $\mathrm{BC}$ 's running time before the mutator makes any progress.

Under severe memory pressure (93MB available RAM), the full bookmarking collector far outstrips the other collectors, achieving almost 0.9 utilization over the 10 second window. At this window size, all of the other collectors have zero utilization. The non- 


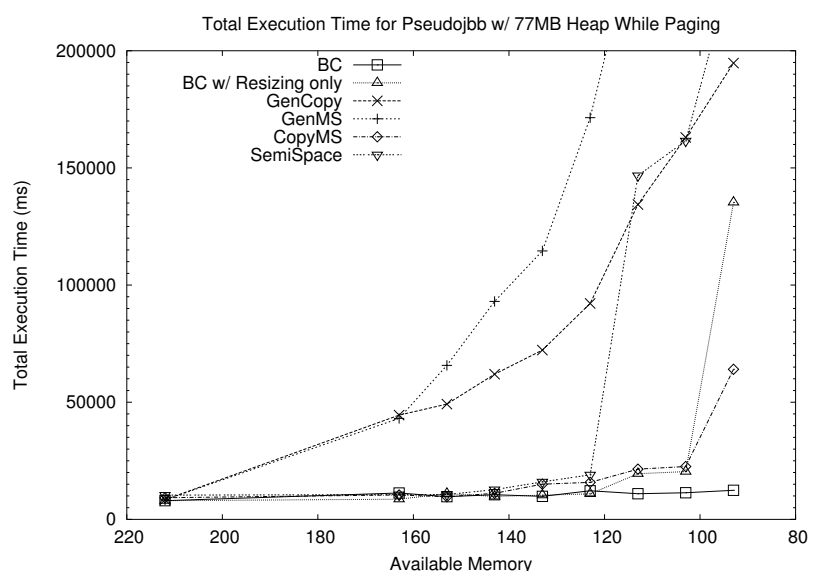

(a) Execution time running pseudoJBB

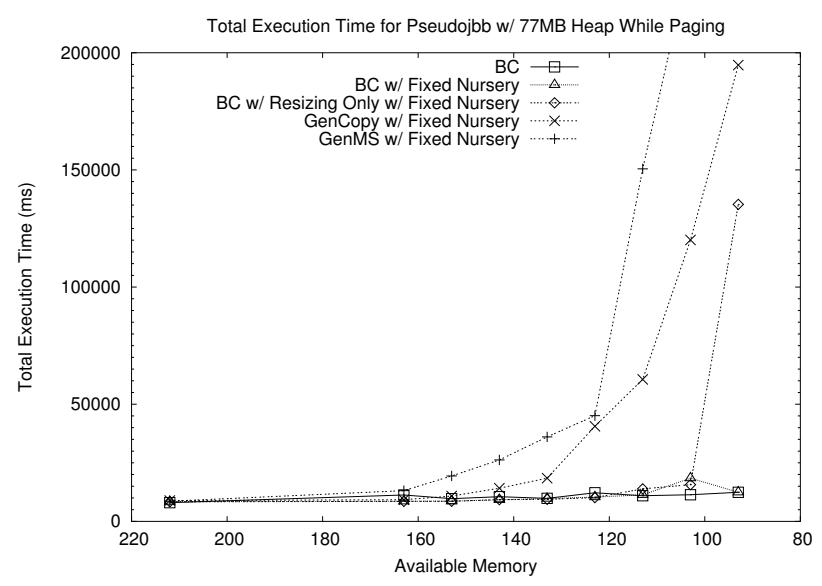

(b) Execution time running pseudoJBB, fixed nursery collectors

Figure 4: Dynamic memory pressure (increasing from left to right). $\mathrm{BC}$ runs up to $4 \mathrm{x}$ faster than the next best collector and up to 41x faster than GenMS. While shrinking the heap can help, BC runs up to 10x faster when also using bookmarks.

BMU for pseudoJBB w/ 77 MB Heap and 143 MB Available

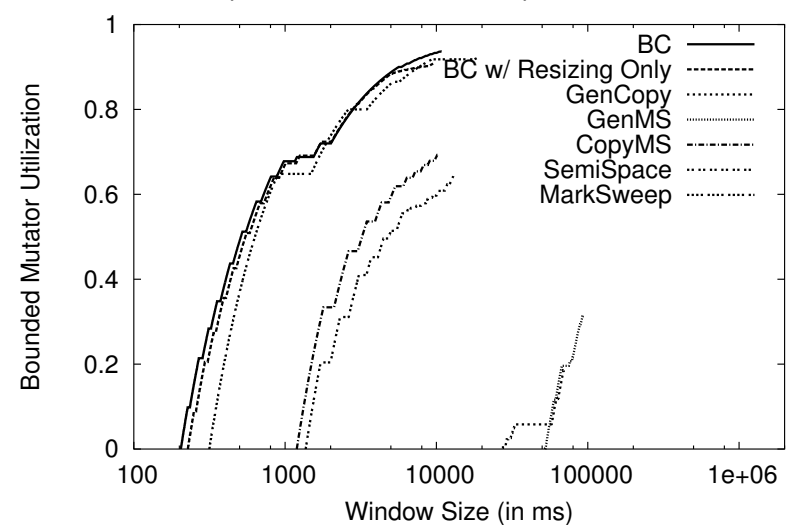

(a) $143 \mathrm{MB}$ available: $\mathrm{BC}$ is largely unaffected by moderate levels of memory pressure.

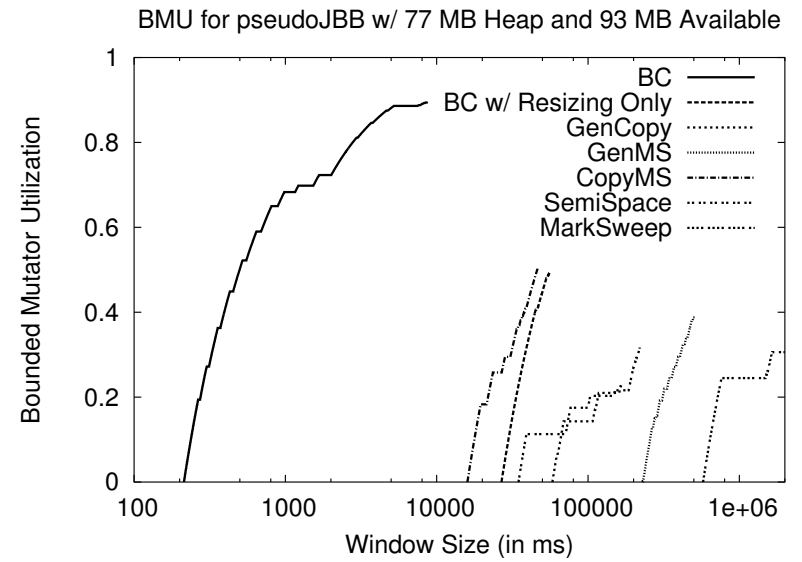

(b) $93 \mathrm{MB}$ available: under heavy memory pressure, bookmarks become increasingly important.

Figure 5: Dynamically increasing memory pressure: bounded mutator utilization curves (curves to the left and higher are better). BC consistently provides high mutator utilization over time. As memory pressure increases (available memory shrinks), the importance of bookmarks to limit garbage collection pauses becomes more apparent.

bookmarking variant of $\mathrm{BC}$ (with resizing only) and CopyMS are the next best collectors, but top out at around 0.5 mutator utilization over a 40 second window. Interestingly, MarkSweep now has the worst utilization, requiring a window size of almost 10 minutes before achieving 0.25 mutator utilization.

We also compared different variants of the bookmarking collector and the generational collectors to tease apart the impact of various strategies on paging.

Fixed-size nurseries: While variable-sized nurseries achieve high throughput, they are generally believed to incur higher paging costs. Figure 4(b) presents execution times for variants of the generational collectors with fixed-size nurseries (4MB). The graph shows that the fixed-nursery variants of the other collectors do reduce paging, but perform just as poorly as the variable-sized generational collectors once their footprint exceeds available memory.

Bookmarking vs. resizing the heap: Initially, $\mathrm{BC}$ is able to avoid paging by simply giving up discardable pages. When memory pressure is relatively low, discarding empty pages is sufficient. As memory pressure increases, however, this approach requires up to 10 times as long to execute as the full bookmarking collec- tor, showing that bookmarking is far more effective than simply shrinking the heap by discarding pages (shown as "BC with resizing only"). The results shown in Figures 2 and 3 demonstrate that bookmarking achieves much higher throughput and lower pause times than resizing the heap.

\section{Multiple JVMs}

Finally, we examine a scenario with two JVMs executing simultaneously. For this experiment, we start two instances of Jikes running the pseudoJBB benchmark and measure total elapsed time and garbage collection pause times. We cannot employ the "compileand-reset" experimental methodology here because compiling the entire application generates too much paging traffic and we cannot "reset" the virtual memory manager's history. Instead, we employ the pseudoadaptive methodology [33,46], which optimizes only the "hottest" methods (as determined from the mean of 5 runs). This methodology minimizes the impact of the compiler as much as possible, and does so deterministically rather than relying on the standard sampling-based approach. Eeckhout et al. report that all of the virtual machines they examined exhibit similar behavior 


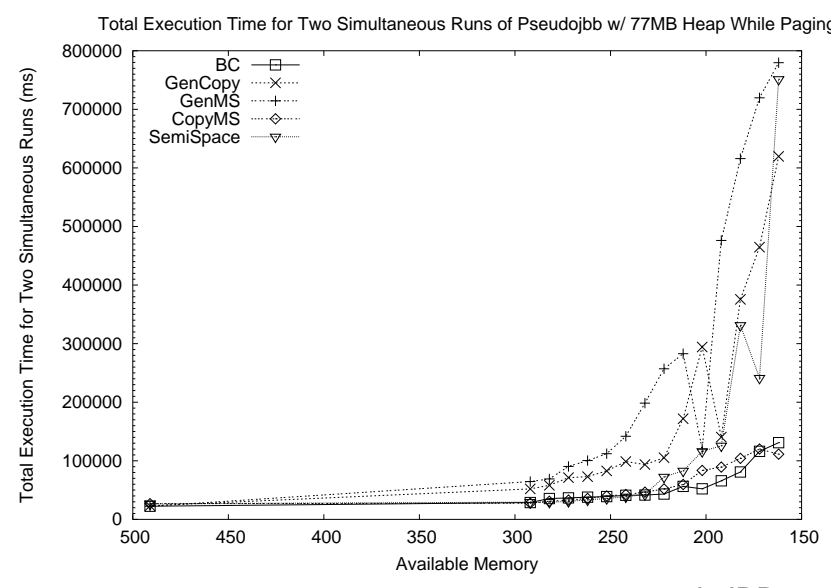

(a) Execution time running two instances of pseudoJBB

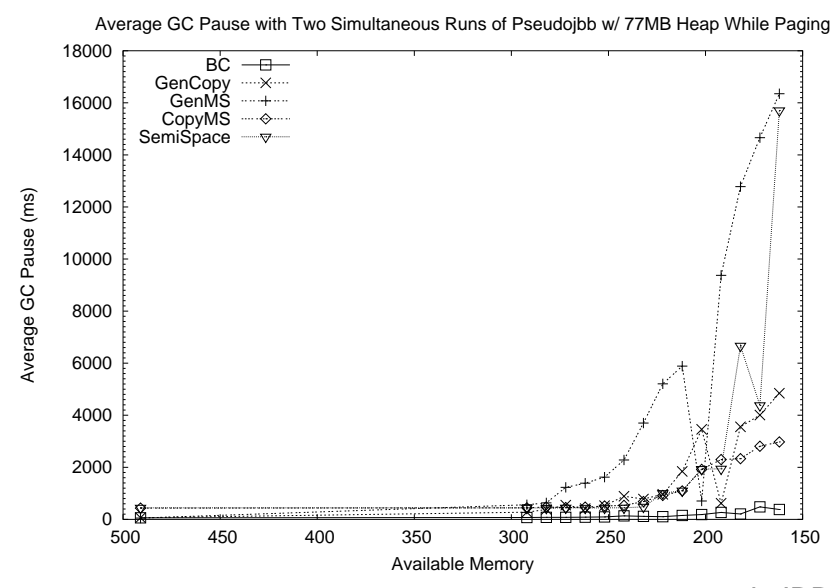

(b) Average GC pause time running two instances of pseudoJBB

Figure 6: Execution time and pause times when running two instances of pseudoJBB simultaneously. Note that the execution times are somewhat misleading, because for the other collectors, paging effectively deactivates one of the instances. Under heavy memory pressure, BC exhibits pause times around $7.5 x$ lower than the next best collector.

for this benchmark, suggesting that the performance impact of the pseudoadaptive compiler here should be minimal [30].

Figure 6 shows the results of executing two instances of pseudoJBB, each with a 77MB heap. While BC performs the best, total elapsed time can be misleading: for all of the other collectors, paging effectively deactivates one of the instances. This allows one of the instances of pseudoJBB to run to completion, at which point the other begins. The average pause time numbers are more revealing. As available memory shrinks, bookmarking shows a gradual increase in average pause times. At the lowest amount of available memory, BC exhibits pause times averaging around $380 \mathrm{~ms}$, while average pause times for CopyMS (the next best collector) reach 3 seconds, nearly an eightfold increase.

\section{Related Work}

Most previous garbage collectors ignore virtual memory altogether, making them VM-oblivious. The literature on such collectors is extensive; Wilson's survey and Jones and Lins' text provide excellent overviews $[34,55]$.

VM-sensitive garbage collectors are designed to limit paging in virtual memory environments. Such collectors either compact memory to reduce the number of pages needed to hold an application's working set $[14,15,21,22,23,31]$, or divide the heap into generations to reduce the need for full-heap collections [9, 17, 38, 40, 43, 51]. Bookmarking collection builds on both: it employs compaction to reduce working set size and uses a nursery generation to improve throughput, but uses bookmarking to avoid collector-induced paging. Bookmarking collection may be thought of as a generalization of generational collection, where the evicted pages comprise a distinct (and dynamically changing) mature-space generation, and bookmarks act as remembered sets.

VM-cooperative garbage collectors receive information from or send information to the virtual memory manager (or both). Moon's ephemeral collector receives signals from the virtual memory manager whenever it evicts a page from main memory [40]. The collector then scans the page and records which generations it contains references to. During GC, Moon's collector then scans only those evicted pages containing pointers to the generation being collected. Unlike Moon's collector, bookmarking collection does not revisit any evicted pages during nursery or full-heap collections.

Cooper et al.'s collector informs the VM of empty memory pages that can be removed from main memory without being written back to disk [26]. BC also identifies and discards empty pages, but as we show in Section 6.3, BC's ability to evict non-empty pages gives it a significant advantage under memory pressure.

Another class of VM-cooperative collectors respond to memory pressure by adjusting their heap size. Alonso and Appel present a collector that consults with the virtual memory manager indirectly (through an "advisor") to shrink the heap after each garbage collection based upon the current level of available memory [6]. MMTk [19] and BEA JRockit [3] can, in response to the live data ratio or pause time, change their heap size using a set of pre-defined ratios. HotSpot [1] can adjust heap size with respect to pause time, throughput, and footprint limits specified as command line arguments. Novell Netware Server 6 [2] polls the virtual memory manager every 10 seconds, and shortens its GC invocation interval to collect more frequently when memory pressure is high. None of these approaches eliminate paging. Yang et al. report on an automatic heap sizing algorithm that uses information from a simulated virtual memory manager and a model of GC behavior to choose a good heap size [57]. Like these systems, BC can shrink its heap (by giving up discardable pages), but it does not need to wait until a full heap garbage collection to do so. $\mathrm{BC}$ also eliminates paging even when the size of live data exceeds available physical memory.

Researchers have leveraged virtual memory hardware to support garbage collection in other ways. Appel, Ellis and Li use virtual memory protection to improve the speed and concurrency of Baker's algorithm [10]. Appel and Li present a variety of ways to use virtual memory to assist garbage collection, including providing an inexpensive means of synchronization, remembering interesting pointers, or saving long-lived data to disk [11]. These uses of virtual memory are orthogonal to this work and to the VMcooperative collectors described above, which use virtual memory cooperation primarily to reduce paging.

Like bookmarking collection, distributed garbage collection algorithms also treat certain references (those from remote systems) as secondary roots. ${ }^{3}$ Distributed GC algorithms typically employ either reference counting or listing. Unlike bookmarking, both of these require substantial additional memory and updates on every

\footnotetext{
${ }^{3}$ See Abdullahi and Ringwood [4] and Plainfossé and Shapiro [42] for excellent recent surveys of this area.
} 
pointer store, while $\mathrm{BC}$ only updates bookmarks when pages are evicted or made resident (relatively rare events).

A number of researchers have focused on the problem of improving application-level locality of reference by using the garbage collector to modify object layouts $[5,25,29,33,36,49,50$, 56]. These studies do not address the problem of paging caused by garbage collection itself, which we identify as the primary culprit. These approaches are orthogonal and complementary to the work presented here.

Finally, bookmarking collection's use of segregated size classes for compaction is similar to the organization used by Bacon et al.'s Metronome collector [12, 13]. Unlike the Metronome, BC uses segregated size classes for mature objects only. BC's copying pass is also quite different. The Metronome sorts pages by occupancy, forwards objects by marching linearly through the pages and continues until reaching a pre-determined size, forwarding pointers later. BC instead copies objects by first marking target pages and then forwarding objects as they are discovered in a Cheney scan. This approach obviates the need for further passes and immediately brings memory consumption to the minimum level.

\section{Future Work}

While our results show that $\mathrm{BC}$ already yields significant performance improvements and robustness under memory pressure, there are several directions in which we would like to advance this work. First, because our modifications to the operating system kernel are straightforward, we would like to incorporate these in other operating systems to verify its generality and expand our range of benchmark applications. We are also interested in applying the bookmarking collection approach to incremental garbage collectors.

The bookmarking collector currently focuses on finding a heap size in which it can run that does not significantly increase memory pressure. We are exploring extensions to the virtual memory manager that will allow BC to cheaply determine when it is appropriate to grow the resident heap [57].

We are considering alternate strategies for selecting victim pages. First, we can prefer to evict pages with no pointers, because these pages cannot create false garbage. For some types of objects, e.g., arrays of doubles, we do not even need to scan the pages to determine that they are free of pointers. We could also prefer to evict pages with as few non-NULL pointers as possible. We have not yet explored these possibilities because we cannot predict the effect on the application of evicting a page that is not the last on the LRU queue (i.e., the one chosen by the virtual memory manager). Choosing to evict such a victim page may lead to more page faults in the application. We are currently developing a more advanced virtual memory manager that will enable us to predict the effect of selecting different victim pages and thus explore the tradeoffs of using more sophisticated eviction strategies. Finally, we are investigating the use of static analyses to provide connectivity or lifetime information to the garbage collector in order to reduce the reliance on pointer summaries.

\section{Conclusion}

The increasing latency gap between disk and main memory means that paging is now intolerable. Garbage collection's reference behavior can cause catastrophic paging. We present bookmarking collection, an algorithm that leverages cooperation between the garbage collector and virtual memory manager to eliminate nearly all paging caused by the garbage collector. When memory pressure is low, the bookmarking collector provides performance that generally matches or slightly exceeds that of the highest throughput collector we tested (GenMS). In the face of memory pressure, BC improves program performance by up to $5 \mathrm{x}$ over the next best gar- bage collector and reduces pause times by $45 x$, improving even more dramatically over GenMS. BC thus provides greater memory utilization and more robust performance than previous garbage collectors.

\section{Acknowledgements}

Emery Berger was supported by NSF CAREER Award number CNS-0347339. Thanks to Sam Guyer, Kathryn McKinley, Eliot Moss, Pritesh Sharma, Yannis Smaragdakis, and Ben Zorn for their comments on drafts of this paper. We are grateful to Scott Kaplan for his assistance in the implementation of our modified Linux memory manager. We are also grateful to IBM Research for making the Jikes RVM system available under open source terms. The MMTk memory management toolkit was particularly helpful.

\section{References}

[1] J2SE 1.5.0 Documentation - Garbage Collector Ergonomics. Available at http://java.sun.com/j2se/1.5.0/docs/ guide/vm/gc-ergonomics.html.

[2] Novell Documentation: NetWare 6 - Optimizing Garbage Collection. Available at http:

//www. novell.com/documentation/index.html.

[3] Technical white paper - BEA weblogic jrockit: Java for the enterprise. Available at http://www.bea.com/content/ news_events/white_papers/BEA_JRockit_wp.pdf.

[4] S. E. Abdullahi and G. A. Ringwood. Garbage collecting the Internet: a survey of distributed garbage collection. ACM Computing Surveys, 30(3):330-373, Sept. 1998.

[5] A.-R. Adl-Tabatabai, R. L. Hudson, M. J. Serrano, and S. Subramoney. Prefetch injection based on hardware monitoring and object metadata. In Proceedings of the 2004 ACM SIGPLAN Conference on Programming Language Design and Implementation (PLDI). ACM, June 2004.

[6] R. Alonso and A. W. Appel. Advisor for flexible working sets. In Proceedings of the 1990 ACM Sigmetrics Conference on Measurement and Modeling of Computer Systems, pages 153-162, Boulder, CO, May 1990.

[7] B. Alpern, C. R. Attanasio, A. Cocchi, D. Lieber, S. Smith, T. Ngo, J. J. Barton, S. F. Hummel, J. C. Shepherd, and M. Mergen. Implementing Jalapeño in Java. In Proceedings of the ACM Conference on Object-Oriented Systems, Languages and Applications, volume 34(10), pages 314-324, Denver, CO, Oct. 1999.

[8] B. Alpern, D. Attanasio, J. J. Barton, M. G. Burke, P. Cheng, J.-D. Choi, A. Cocchi, S. J. Fink, D. Grove, M. Hind, S. F. Hummel, D. Lieber, V. Litvinov, M. Mergen, T. Ngo, J. R. Russell, V. Sarkar, M. J. Serrano, J. Shepherd, S. Smith, V. C. Sreedhar, H. Srinivasan, and J. Whaley. The Jalapeño virtual machine. IBM Systems Journal, 39(1), Feb. 2000.

[9] A. W. Appel. Simple generational garbage collection and fast allocation. Software Practice and Experience, 19(2):171-183, 1989.

[10] A. W. Appel, J. R. Ellis, and K. Li. Real-time concurrent collection on stock multiprocessors. ACM SIGPLAN Notices, 23(7):11-20, 1988.

[11] A. W. Appel and K. Li. Virtual memory primitives for user programs. ACM SIGPLAN Notices, 26(4):96-107, 1991.

[12] D. F. Bacon, P. Cheng, and V. Rajan. Controlling fragmentation and space consumption in the Metronome, a real-time garbage collector for Java. In ACM SIGPLAN 2003 Conference on Languages, Compilers, and Tools for Embedded Systems (LCTES'2003), pages 81-92, San Diego, CA, June 2003. ACM Press.

[13] D. F. Bacon, P. Cheng, and V. Rajan. A real-time garbage collecor with low overhead and consistent utilization. In Conference Record of the Thirtieth Annual ACM Symposium on Principles of Programming Languages, ACM SIGPLAN Notices, New Orleans, LA, Jan. 2003. ACM Press.

[14] H. D. Baecker. Garbage collection for virtual memory computer systems. Communications of the ACM, 15(11):981-986, Nov. 1972.

[15] H. G. Baker. List processing in real-time on a serial computer. Communications of the ACM, 21(4):280-94, 1978.

[16] E. D. Berger, K. S. McKinley, R. D. Blumofe, and P. R. Wilson. Hoard: A scalable memory allocator for multithreaded applications. 
In ASPLOS-IX: Ninth International Conference on Architectural Support for Programming Languages and Operating Systems, pages 117-128, Cambridge, MA, Nov. 2000.

[17] P. B. Bishop. Computer Systems with a Very Large Address Space and Garbage Collection. PhD thesis, MIT Laboratory for Computer Science, May 1977.

[18] S. M. Blackburn, P. Cheng, and K. S. McKinley. Myths and reality: The performance impact of garbage collection. In Sigmetrics Performance 2004, Joint International Conference on Measurement and Modeling of Computer Systems, New York, NY, June 2004.

[19] S. M. Blackburn, P. Cheng, and K. S. McKinley. Oil and water? High performance garbage collection in Java with MMTk. In ICSE 2004, 26 th International Conference on Software Engineering, Edinburgh, May 2004.

[20] S. M. Blackburn and K. S. McKinley. Ulterior reference counting: Fast garbage collection without a long wait. In OOPSLA [41].

[21] D. G. Bobrow and D. L. Murphy. Structure of a LISP system using two-level storage. Communications of the ACM, 10(3):155-159, Mar. 1967.

[22] D. G. Bobrow and D. L. Murphy. A note on the efficiency of a LISP computation in a paged machine. Communications of the ACM, 11(8):558-560, Aug. 1968.

[23] C. J. Cheney. A non-recursive list compacting algorithm. Communications of the ACM, 13(11):677-8, Nov. 1970.

[24] P. Cheng and G. Belloch. A parallel, real-time garbage collector. In Proceedings of SIGPLAN 2001 Conference on Programming Languages Design and Implementation, ACM SIGPLAN Notices, pages 125-136, Snowbird, Utah, June 2001. ACM Press.

[25] T. M. Chilimbi and J. R. Larus. Using generational garbage collection to implement cache-conscious data placement. In Proceedings of the First International Symposium on Memory Management, volume 34(3), pages 37-48, Vancouver, BC, Canada, Oct. 1998.

[26] E. Cooper, S. Nettles, and I. Subramanian. Improving the performance of SML garbage collection using application-specific virtual memory management. In Conference Record of the 1992 ACM Symposium on Lisp and Functional Programming, pages 43-52, San Francisco, CA, June 1992. ACM Press.

[27] S. P. E. Corporation. Specjbb2000. Available at http: //www . spec.org/jbb2000/docs/userguide. html.

[28] S. P. E. Corporation. Specjvm98 documentation, Mar. 1999.

[29] R. Courts. Improving locality of reference in a garbage-collecting memory management-system. Communications of the ACM, 31(9):1128-1138, 1988.

[30] L. Eeckhout, A. Georges, and K. D. Bosschere. How Java programs interact with virtual machines at the microarchitectural level. In OOPSLA [41], pages 169-186.

[31] R. R. Fenichel and J. C. Yochelson. A Lisp garbage collector for virtual memory computer systems. Communications of the ACM, 12(11):611-612, Nov. 1969.

[32] M. Hertz and E. D. Berger. Automatic vs. explicit memory management: Settling the performance debate. Technical report, University of Massachusetts, Mar. 2004.

[33] X. Huang, S. M. Blackburn, K. S. McKinley, J. E. B. Moss, Z. Wang, and $P$. Cheng. The garbage collection advantage: Improving program locality. In Proceeding of the ACM Conference on Object-Oriented Systems, Languages and Applications, Vancouver, BC, Canada, Oct. 2004.

[34] R. E. Jones and R. Lins. Garbage Collection: Algorithms for Automatic Dynamic Memory Management. Wiley, Chichester, July 1996.

[35] S. F. Kaplan. In-kernel RIG: Downloads. Available at http://www.cs.amherst.edu/ sfkaplan/research/ rig/download.

[36] M. S. Lam, P. R. Wilson, and T. G. Moher. Object type directed garbage collection to improve locality. In Proceedings of International Workshop on Memory Management, volume 637 of Lecture Notes in Computer Science, St Malo, France, 16-18 Sept. 1992. Springer-Verlag.

[37] D. Lea. A memory allocator. http://gee.cs.oswego.edu/dl/html/malloc.html, 1997. Available at http://gee.cs.oswego.edu/dl/html/malloc.html.
[38] H. Lieberman and C. E. Hewitt. A real-time garbage collector based on the lifetimes of objects. Communications of the ACM, 26(6):419-429, 1983.

[39] T. F. Lim, P. Pardyak, and B. N. Bershad. A memory-efficient real-time non-copying garbage collector. In Proceedings of the First International Symposium on Memory Management, volume 34(3), pages 118-129, Vancouver, BC, Canada, Oct. 1998.

[40] D. A. Moon. Garbage collection in a large LISP system. In G. L. Steele, editor, Conference Record of the 1984 ACM Symposium on Lisp and Functional Programming, pages 235-245, Austin, TX, Aug. 1984. ACM Press.

[41] OOPSLA'03 ACM Conference on Object-Oriented Systems, Languages and Applications, ACM SIGPLAN Notices, Anaheim, CA, Nov. 2003. ACM Press.

[42] D. Plainfossé and M. Shapiro. A survey of distributed garbage collection techniques. In Proceedings of the International Workshop on Memory Management, volume 986 of Lecture Notes in Computer Science, Kinross, Scotland, Sept. 1995. Springer-Verlag.

[43] J. H. Reppy. A high-performance garbage collector for Standard ML. Technical memorandum, AT\&T Bell Laboratories, Murray Hill, NJ, Dec. 1993.

[44] D. T. Ross. The AED free storage package. Communications of the ACM, 10(8):481-492, Aug. 1967.

[45] N. Sachindran and J. E. B. Moss. MarkCopy: Fast copying GC with less space overhead. In Proceedings of the ACM Conference on Object-Oriented Systems, Languages and Applications, Anaheim, CA, Nov. 2003.

[46] N. Sachindran, J. E. B. Moss, and E. D. Berger. MC ${ }^{2}$ : High-performance garbage collection for memory-constrained environments. In Proceedings of the ACM Conference on Object-Oriented Systems, Languages and Applications, Vancouver, BC, Canada, Oct. 2004.

[47] P. Savola. LBNL traceroute heap corruption vulnerability. http: / / www. securityfocus. com/bid/1739.

[48] Software Verification, Ltd. Memory Validator - Garbage Collectors. Available at http://softwareverify.com/ memoryValidator/garbageCollectors.html.

[49] D. Stefanović, K. S. McKinley, and J. E. B. Moss. Age-based garbage collection. In Proceedings of the ACM Conference on Object-Oriented Systems, Languages and Applications, volume 34(10), pages 370-381, Denver, CO, Oct. 1999.

[50] G. Tong and M. J. O'Donnell. Leveled garbage collection. Journal of Functional and Logic Programming, 2001(5):1-22, May 2001.

[51] D. M. Ungar. Generation scavenging: A non-disruptive high performance storage reclamation algorithm. In Proceedings of the ACM/SIGSOFT/SIGPLAN Software Engineering Symposium on Practical Software Development Environments, volume 19(5), pages 157-167, Apr. 1984.

[52] R. van Riel. rmap VM patch for the Linux kernel. Available at http: / / www. surriel.com/patches/.

[53] D. A. Wheeler. SLOCcount. Available at http: //www. dwheeler. com/sloccount.

[54] P. R. Wilson. Uniprocessor garbage collection techniques. In Proceedings of the International Workshop on Memory Management, volume 637 of Lecture Notes in Computer Science, St Malo, France, 16-18 Sept. 1992. Springer-Verlag.

[55] P. R. Wilson, M. S. Johnstone, M. Neely, and D. Boles. Dynamic storage allocation: A survey and critical review. In Proceedings of the International Workshop on Memory Management, volume 986 of Lecture Notes in Computer Science, pages 1-116, Kinross, Scotland, Sept. 1995. Springer-Verlag.

[56] P. R. Wilson, M. S. Lam, and T. G. Moher. Effective static-graph reorganization to improve locality in garbage collected systems. ACM SIGPLAN Notices, 26(6):177-191, 1991.

[57] T. Yang, E. D. Berger, M. Hertz, S. F. Kaplan, and J. E. B. Moss. Automatic heap sizing: Taking real memory into account. In Proceedings of the 2004 ACM SIGPLAN International Symposium on Memory Management, Nov. 2004.

[58] B. Zorn. The measured cost of conservative garbage collection. Software Practice and Experience, 23:733-756, 1993. 